\title{
DESIGN OF HYBRID COMPONENTS JOINING ZONE THROUGH SENSITIVITY ANALYSIS
}

\author{
Siqueira, Renan; Shugar, Sean; Mozgova, Iryna; Lachmayer, Roland \\ Leibniz University Hannover, Institute for Product Development
}

\begin{abstract}
Multi-material structures are a trending topic for the industry. With a high application potential, such as lightweight or extended life cycle, different manufacturing technologies are further developed for this intent. One of these technologies is Tailored Forming, a process-chain capable of joining different metals and creating massive hybrid components. In parallel to this development, new challenges rise for design, which has the responsibility of finding an optimal use of this technology and produce higherperformance products. However, this task cannot be solved by conventional engineering approach, since strong manufacturing constraints are involved and a lack of understanding about the joining zone formed between the materials still exists. To fill this gap, the objective of this study is to analyse the influence of the joining zone design over the structure behaviour and establish a suitable design method. For that, a computer-aided environment was constructed and a parametric sensitivity analysis was executed, taking a hybrid shaft as example. At the end, the simulation's results allowed a multi-objective optimisation and were able to generate first design guidelines.
\end{abstract}

Keywords: Computer Aided Design (CAD), Design methods, Lightweight design, Multi-material, Sensitivity Analysis

\section{Contact:}

da Silva de Siqueira, Renan

Leibniz University Hannover

IPEG

Germany

siqueira@ipeg.uni-hannover.de

Cite this article: Siqueira, R., Shugar, S., Mozgova, I., Lachmayer, R. (2019) 'Design of Hybrid Components Joining Zone through Sensitivity Analysis', in Proceedings of the 22nd International Conference on Engineering Design (ICED19), Delft, The Netherlands, 5-8 August 2019. DOI:10.1017/dsi.2019.276 


\section{INTRODUCTION}

In recent years, steadily increasing demands on technical components have been observed. Above all, the automotive industry will need more and more components in the future that simultaneously conserve resources, protect the climate and improve performance. A vehicle mass reduced by $100 \mathrm{~kg}$, for example, leads to a reduction in fuel consumption by about 0.3 to $0.51 / 100 \mathrm{~km}$ and $\mathrm{CO}_{2}$ emissions by about 8.5 to $14 \mathrm{~g} / \mathrm{km}$. However, many vehicles have seen an averaged increase of $100 \mathrm{~kg}$ in weight per decade, due, among other things, to the rise of safety and comfort requirements in automobile construction (Lauter 2014). To counteract this trend, innovative and above all holistic lightweight construction approaches are required. While materials such as steel, aluminium and plastics are individually developed further, more and more engineers and manufacturers are looking for the optimum in hybrid or multi-material construction (Roper et al., 2018, Siqueira et al., 2018).

One of these new hybrid construction methodologies is Tailored Forming, a process-chain that involves a primary connection between work-pieces from different metals, followed by other forming and machining processes (Figure 1) (Behrens et al., 2017). The result of this technology is a massive hybrid component, in which the material distribution allows an adjusted local functionality. With that, a more reliable and effective product can be achieved, with properties such as lightweight, reduced volume, extended life-cycle or adapted function (Brockmoeller et al., 2017). Although a higher number of design degrees of freedom is a wanted feature for solution exploration in the future of engineering, such hybrid designs are not covered by classical construction methods, requiring the use of modern approaches to deal with the new challenges. In the case of Tailored Forming, a specific method has to be introduced to deal with the manufacturing limitations and the design of the joining zone between the two materials.

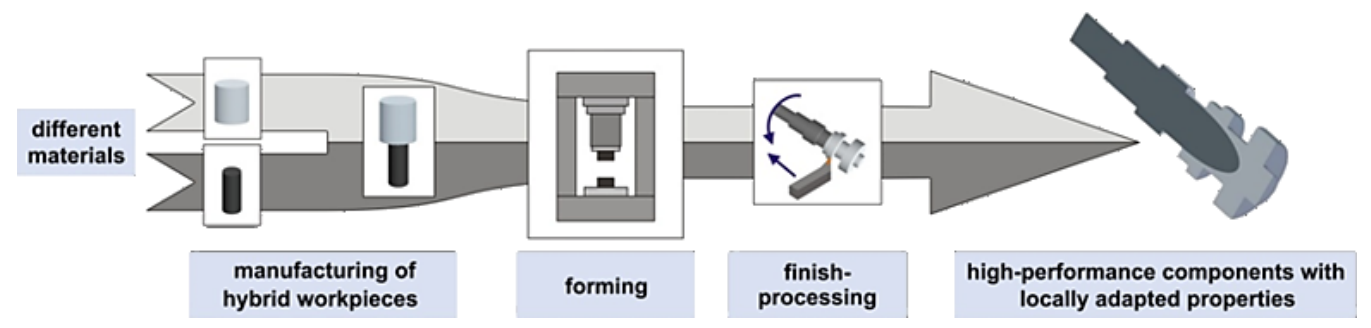

Figure 1. Representation of the process-chain of tailored forming (Behrens et al., 2017)

There is still a lack of understanding and practical experience about the interaction of different materials in a single component. For instance, research about the modelling of the joining zone between different metals is still under development (Behrens et al., 2018). In the design field, this joining zone represents also an additional geometry interface that must specified. For that, the influence that a change in the joining zone might have in the performance of the structure must be measured and formulated. As seen in (Siqueira et al., 2019), a preliminary analysis about this influence using a parametric approach was executed, where a clear dependency between design parameters and requirements was shown. However, this study did not deliver a precise and practical method on how to construct the parametric model and how to prioritise the parameters in the manufacturing process.

With that, the method performed in this paper starts with a parametric construction, followed by a finite element (FE) analysis and construction of approximation functions, and finishing with a sensitivity analysis of the parameters. For that, it is taken the example of a hybrid shaft made of steel and aluminium alloy, component that can be currently manufactured by Tailored Forming and has a vast application. The objective is to establish a guideline on how future engineers should approach such designs in order to achieve the desired higher performance. At the end, a multi-optimisation analysis was performed, to point out the advantages of the method applied.

\section{METHODS}

\subsection{Response surface methodology (RSM)}

The main costs of obtaining information about the mechanical system behaviour of components or assemblies are due to FE solver calls (Harzheim 2014). Therefore, efforts are made to keep the number of FE analyses at a minimum. This is possible by using approximations. Approximations replace expensive 
analyses and have the task of approximating the system response satisfactorily precisely in the design space. Response surface methodology (RSM) is a collection of statistical and mathematical techniques useful for developing, improving and optimising processes (Box and Draper 2007). It has also important applications in the design, developement and formulation of new products, as well as in the improvement of existing product designs (R. Myers and Anderson-Cook 2009).

As a rule, there are no analytical expressions for the system responses in structure optimisation. In practice, the system responses are therefore replaced by metamodels (Harzheim 2014). Metamodels are global approximations that describe the system response in the entire design space. The real and unknown system response is replaced by a response surface. Using the least squares method, the approximation is adjusted so that the sum of the differences between the approximated values and the actual values globally across the design space is a minimum. To keep the amount of solver runs at a minimum, the design space is sampled through stochastic sampling methods such as the Monte Carlo or Latin Hypercube Sampling (LHS).

It is important to note that there are various approximation methods that can be applied for a case. Examples include isotropic kriging and moving least square regression (Dynardo GmbH 2018). However, the polynomial approach provides a good mixture of accuracy and simplicity and is therefore very popular and often used (R. Myers and Anderson-Cook 2009). Often the first-order approach can well describe a system response. However, if non-linearities or strong interactions occur, the second-order polynomial approach or other equations can be used instead.

\subsection{Sensitivity analysis (ANOVA)}

By definition, sensitivity analysis is the study of how the uncertainty in the output of a model can be apportioned, qualitatively or quantitatively, to different sources of variation in the input of a model (Saltelli 2008). A sensitivity analysis can serve a number of useful purposes in the economy of modelling, since it is used as an effective pre-processing tool for engineering design and optimisation (Ullman 2010). It helps the engineer to understand the design and the relationships between input and output variables by detecting technical errors in the model. So, by analysing the results of a sensitivity study, it is possible to simplify a model, since significant parameters and correlations can be identified and filtered (Saltelli 2008). The identification of the most important input parameters establishes priorities for research and leads to the recognition of design improvements.

Global methods for sensitivity analysis examine the influence of the model input variables on the model response variables in a specific area. The output variables to be optimised describe the design space of the model, which is defined by lower and upper limits. In theory, there are several ways to calculate global sensitivities, such as the analysis of variance (ANOVA). Variance-based sensitivity analyses are implemented in most optimisation programs (Harzheim 2014). In contrast to the local sensitivity method, the variation of the response variables is assigned to the variation of the input variables in the global variant-based method.

Global methods have several advantages. On the one hand, they are insensitive to non-linearities. On the other hand, in addition to individual sensitivities, they also take into account interactions between several input parameters. A distinction is therefore made between first-order effects, which describe the influence of an input variable alone, and higher-order effects, which describe interactions between the input variables. To quantify these effects, the Sobol indices were introduced. Sobol indices of first order describe the variance of the conditional expectation of the output given the value of an output, normalised by the total variance (Saltelli 2008). Higher order Sobol indices characterise the resulting variance resulting from the combination of several variables.

\subsection{Multiobjective optimisation}

Optimisation is a strategy that is applied in many areas of engineering. As computer performance continues to improve, the focus is shifting towards virtual product development. The basic goal of mathematical optimisation is to find the global minimum of an objective function $\mathbf{F}(\mathbf{x})$ (Breitkopf and Coelho 2010). In this case the goal of the optimisation would be to find a vector $\mathbf{x}$ so that equation 2.21 is fulfilled. Vector $\mathbf{x}$ consists of the design or decision variables $x_{i}$ which are defined by their lower and upper bounds $x_{l}$ and $x_{u}$ in the design or decision space. In practice, however, restrictions supplement 
the optimisation problem (Harzheim 2014). Restrictions are boundary conditions such as manufacturing constraints and thus limit the solution area. Inequality restrictions $\mathbf{G}(\mathbf{x})$ indicate that objectives or parameters should be below or above a certain value.

$$
\begin{aligned}
& \mathbf{F}(\mathbf{x})=\left[f_{1}(x), f_{2}(x), \ldots, f_{M}(x)\right] \rightarrow \min \\
& \mathbf{G ( x )}=\left[g_{1}(x), g_{2}(x), \ldots, g_{J}(x)\right] \geq 0
\end{aligned}
$$

Optimisation methods for the solution of individual goals are for example gradient-based methods or the Adaptive Response Surface Method (Dynardo GmbH 2018). For these optimisation problems, where there is only one goal, there is usually a single global minimum and therefore a clear solution. In practice, however, several objectives are aimed at, such as reducing the mass with a simultaneous reduction of the stress (A. Lopez Jaimes and Coello 2001). In this case one speaks of a multiobjective optimisation, where several objective functions $f(x)$ are found. Here it is not specifically the design space that is considered, but the objective space. The objective space compares the respective goals and simplifies the recognition of relationships. If the parameter combinations $\mathbf{x}$ are transferred to the objective space as points $\mathbf{z}$, the result is a point cloud as shown in Figure 2a.

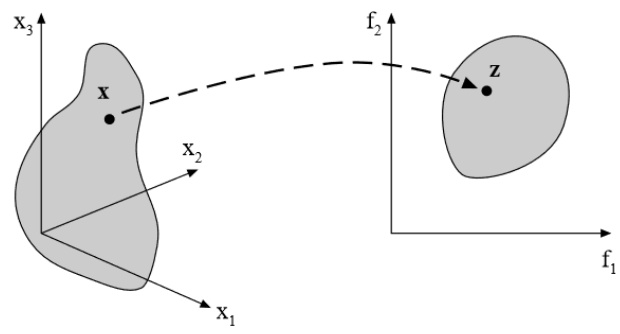

(a) Design space (left) and objective space (right)

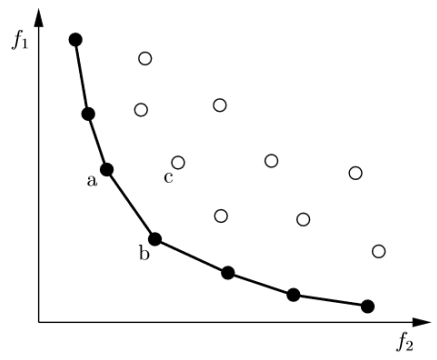

(b) Pareto front

Figure 2. Pareto optimisation (Dynardo GmbH 2018)

The point cloud describes the solution space of the optimisation. If one takes a closer look at the cloud of design points, one sees points on the outermost edge. In this case, each point represents a different design with different input parameters. Nevertheless, not all solution vectors $\mathbf{z}$ are among the best. In most cases, outputs such as mechanical stress, strain or mass should be minimised.

In the case of minimising the two objective functions $f_{1}$ and $f_{2}$, the design points of the point cloud closest to the origin of the objective space are the optimal design points. Figure $2 \mathrm{~b}$ shows the outermost edge of the point cloud, which is called Pareto front. It can be seen that the problem to be optimised usually has several, in this case two, conflicting goals. Therefore, there is no unique optimal solution, but a group of best possible solutions. This, together with the sensitivity analysis, helps with the identification of the most important parameters, as will be seen following.

\section{RESULTS AND DISCUSSION}

In the following, the implementation of the methodology is explained. Three programs were coupled with each other. The realisation of the three-dimensional CAD models was done with Autodesk Inventor. A bidirectional interface with Ansys Workbench allowed the determination of mechanical system responses of the hybrid structure using a finite element analysis without a file conversion of the CAD model. A further advantage is that the parametrisation of the geometry could be retained and imported into the Ansys Workbench parameter manager. The interface was supplemented by the CAE environment OptiSlang from Dynardo (Figure 3) (Dynardo GmbH 2018).

OptiSlang is a software that allows parametric sensitivity analyses and multiobjective optimisations to be performed in interaction with FEM software. The complete integration of OptiSLang within Ansys Workbench and the CAD/FEM interface enabled a full automated computer-aided environment. With that, the generation of parametrised geometries via stochastic evaluations and LHS approach, the construction of the system responses equations and the sensitivity analysis were all executed within one single plattform. To exemplify the method, the design of a hybrid shaft is presented in the next sections. 


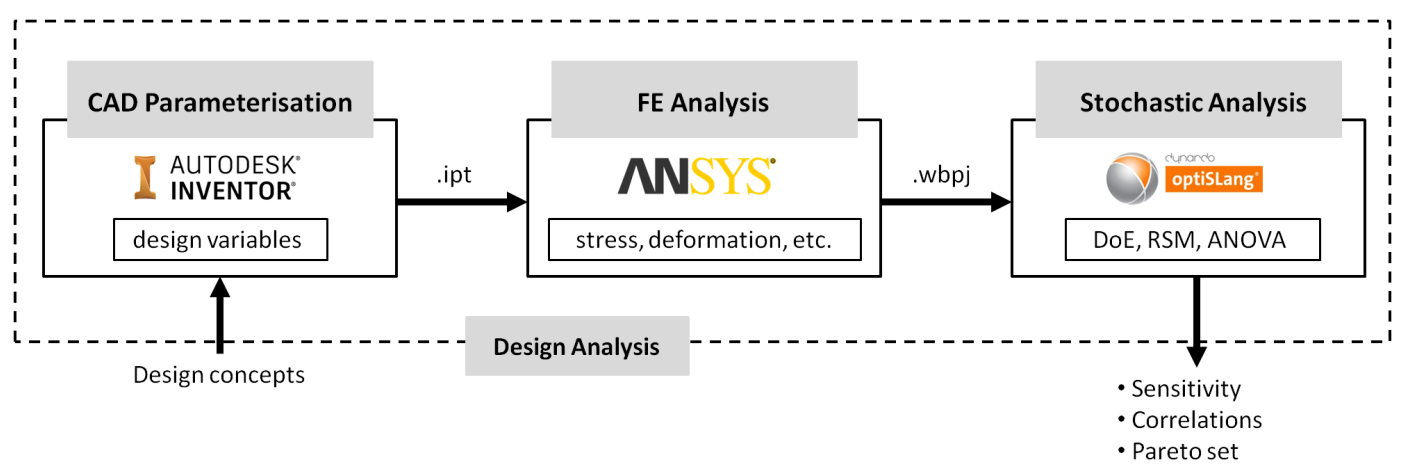

Figure 3. Workflow of the design analysis

\subsection{Task definition}

The workflow created will be here applied for a hybrid shaft manufactured by Tailored Forming and made of steel and aluminium alloy. This shaft is manufactured with a serial connection of the two different metals and later submitted to a forming process under high temperatures. This creates a joining zone between the materials that can be designed in different ways. This design was explored for an specific load case, where a bending force $F$ and a moment $M$ is present. Here, since only linear elastic analysis is under investigation, the magnitude of these forces are not important, but the proportion between them. Based on that, the relation used was a bending force of $100 \mathrm{~N}$ for each $1 \mathrm{Nm}$ of moment. Figure 4 shows the shape of this shaft, main dimensions, position of the joining zone and loads.

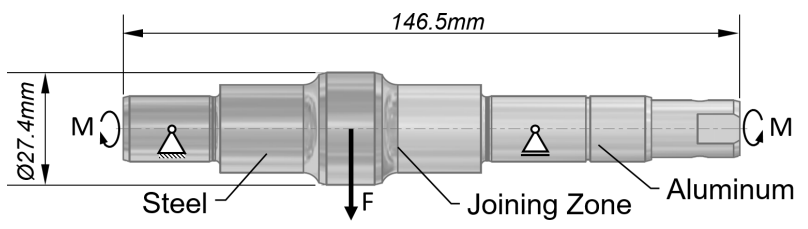

Figure 4. Hybrid shaft case under analysis

The central task of the study is to check the geometry of the joining zone for its mechanical system responses. Two parametrisation concepts were used to dimension the geometry, keeping the position of the joining zone at the same point. Design A was described with the same two parameters from Siqueira et al., (2019), which are variables of a function and thus describe the rotational symmetric contour. For further comparison, Design B was dimensioned with 5 conventional parameters, as seen in Figure $5 \mathrm{~b}$.

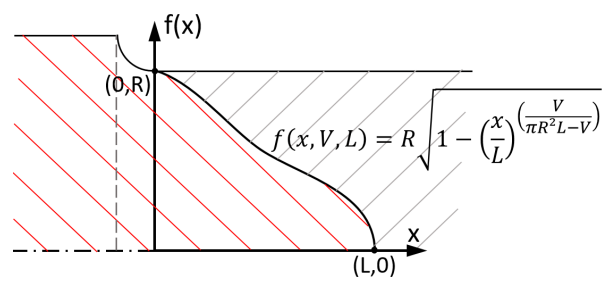

(a) Designs A

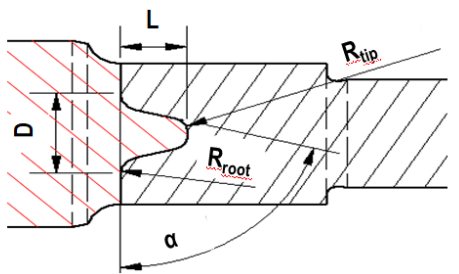

(b) Designs B

Figure 5. Design concepts

In the following, the results of simulations for both results will be presented. Since no fixed load values are being analysed and the system is linear, the outputs will present no scale or magnitude, but relative values.

\subsection{Design A}

The two parameters of design A represent the volume and the length of the joining zone. If one evaluates the response surfaces of the most important system responses of design A, one recognises linear and quadratic relationships within the design space. Figure 6 shows the design space strained by the 
two design parameters together with the maximum deformation and the maximum von Mises stress as system responses.

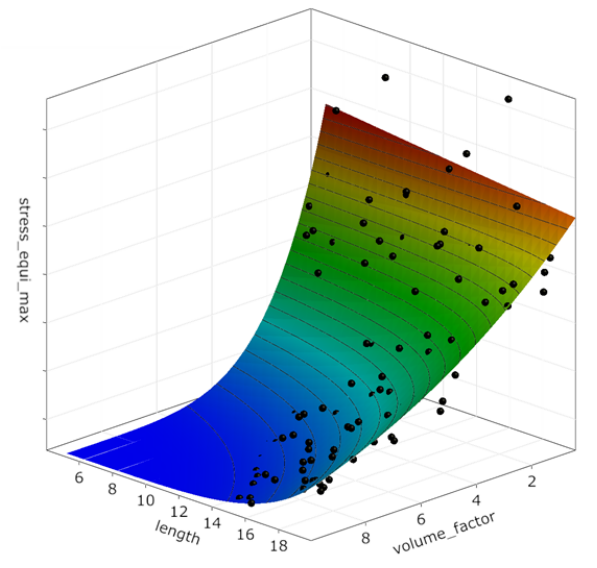

(a) Maximum equivalent stress

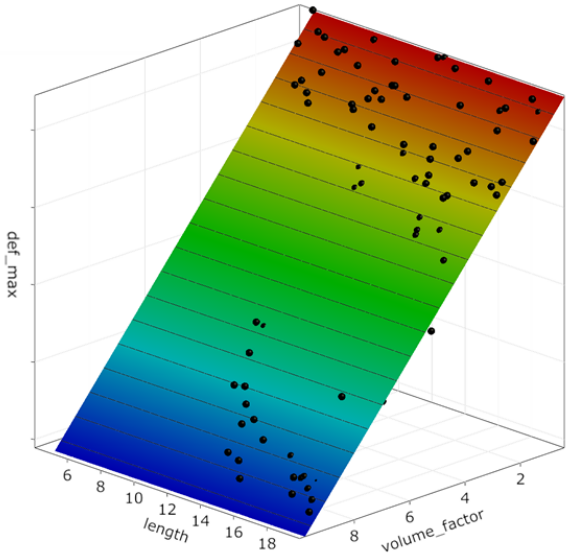

(b) Maximum deformation

Figure 6. Metamodels of design A

Both system responses were well approximated with a polynomial approach of the metamodel. The black points distributed in the design space are the solver call points of the FEA determined by the LHS. The metamodels are able to map the interpolation points well within the design space, which is why the resulting analytical expressions can be used for further purposes, such as multiobjective optimisation. It can be seen that the deformation as well as the maximum stress react very sensitively to the variation of the volume. At the same time it can be observed that the length has very little to no influence on the system responses.

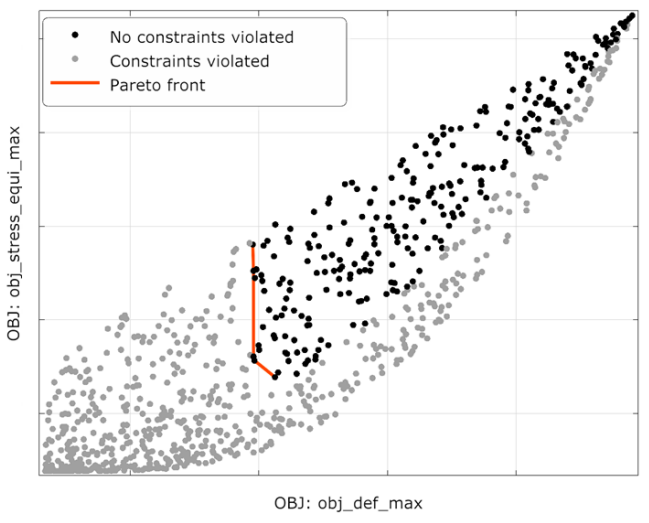

(a) Pareto plot

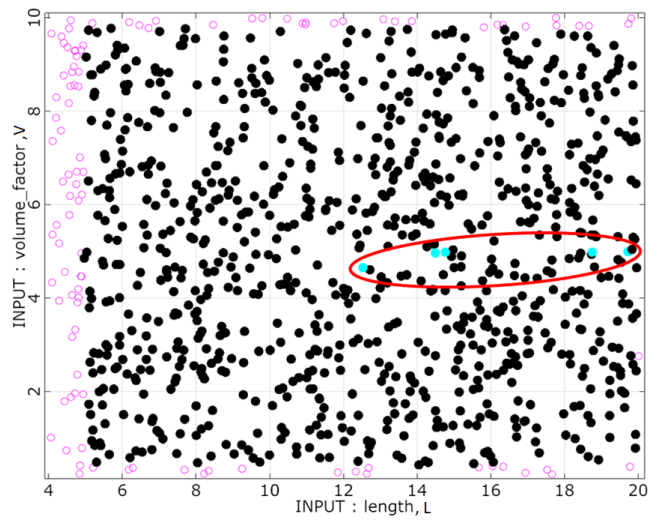

(b) Optimal designs

Figure 7. Multiobjective optimisation of design A

If a multiobjective optimisation is performed in which the objectives are to minimise the maximum stresses while simultaneously minimising the deformation, a Pareto plot is obtained. The Pareto plot in Figure 7a shows the design points generated on the response surface as a function of the system responses. If restrictions are added, e.g. manufacturing restrictions, a Pareto front is visible. It can be seen that the Pareto front is not only dependent on the quality of the metamodel, but also strongly influenced by the accuracy of the restrictions. If one transfers the determined optimal design points of the Pareto front into the design space, one finds a confirmation of the conclusions of the response surfaces. Figure $7 \mathrm{~b}$ shows the design space of design A with the design points generated by the metamodels. The violet design points are failed designs and are due to conflicting parametrisation. If the optimal design points within the pareto front are marked in blue, a strong dependence on the volume of the joining zone can be seen. While the length varies very strongly between $12 \mathrm{~mm}$ and $20 \mathrm{~mm}$, the optimal solutions 
converge at a volume of about $5 \mathrm{~cm}^{3}$. This confirms the highly sensitive influence of the volume on the system responses as well as the independence from the length.

\subsection{Design $B$}

For design B, good approximations for the system responses can also bet obtained. Figure 8 shows the same system responses, such as the maximum equivalent stress and maximum deformation, depending on the three most influential design parameters of design B.

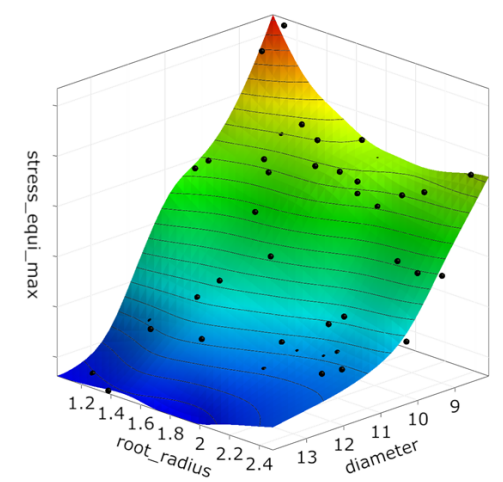

(a) Maximum equivalent stress

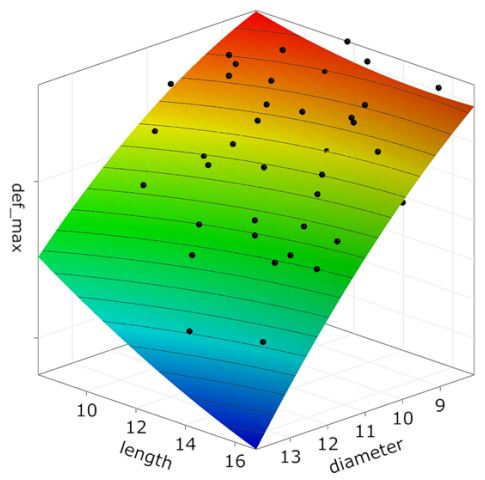

(b) Maximum deformation

Figure 8. Metamodels of design B

The metamodels are approximated by a polynomial approach (Figure $8 \mathrm{~b}$ ) on the one hand and by the Moving Least Squares method (Figure 8a) on the other hand and are able to represent the determined points well. In both diagrams a dependency of the system response of both parameters, which span the respective design space, can be recognised. At the same time, a local dependency of the parameters can be observed. Both response surfaces are non-linear and indicate correlating effects between design parameters. Figure 8a shows a strong influence of the radius on small diameters, while on large diameters the influence of the radius disappears almost completely. Similar to the maximum equivalent stress, correlating effects between length and diameter can be seen with the maximum deformation (Figure 8b). For large diameters, the length has a maximum influence on the global system response. The influence, by varying the length, is negligibly small for small diameters.

\subsection{Discussion}

The correlation- and variance-based sensitivity analysis show a strong dependence of system responses on volume for both designs. Design A has the advantage that it can be designed flexibly using only two parameters to fully map the design space. However, the effects within the system responses are more complicated to identify and quantify through the mathematical relationship of the design variables with many important parameters of the surface. These effects can be more easily seen in design B, where the root radius $R_{\text {root }}$ was parametrised and is also responsible for the variance of the maximum equivalent stress. The higher number of design variables results in several design spaces, which complicate sensitivity analyses and optimisation procedures at a certain point (curse of dimensionality). However, the effects are more accessible to quantify due to the higher parametrisation.

In summary, it can be said that the root radius $R_{\text {root }}$ and the initial diameter $D$ of the joining zone are the most sensitive design parameters, while the total length $L$, which is responsible for correlating effects, gains influence for certain parameter combinations. These three parameters are therefore the most important design parameters to be considered when designing such a hybrid shaft.

\section{CONCLUSION}

The example presented was of a simple component, but such valuable results cannot be clearly obtained in more complicated geometries, in which such analysis method can provide essential information for the design development. Furthermore, the multi-objective optimization was able to expand the understanding of the parameters and confirm the results of the sensitivity analysis. With that, we conclude 
that the design analysis based on an automated association of CAD and FEM software with stochastic tools is well suited for parametric and sensitivity studies. The proposed method provide the engineers a flexible and robust tool that offers a better understanding of such unusual scenario and establish guidelines for the design of a hybrid component. This shows a present tendency in engineering design, where interdisciplinary and modular platforms are preferred in favour of a higher responsibility with future products performance.

\section{REFERENCES}

A. Lopez Jaimes, S. Z. M. and Coello, C. A. C. (2001), Optimization in Polymer Processing, Nova Science Publisher, Inc., chapter An Introduction to Multiobjective Optimization Techniques.

Behrens, B.-A., Bouguecha, A., Moritz, J., Bonk, C., Stonis, M., Klose, C., Blohm, T., Chugreeva, A., Duran, D., Matthias, T., Golovko, O., Thuerer, S. E. and Uhe, J. (2017), 22. umformtechnisches kolloquium, in "Aktuelle Forschungsschwerpunkte in der Massivumformung", Hannoversches Forschungsinstitut fuer Fertigungsverfahren.

Behrens, B.-A., Chugreev, A. and Matthias, T. (2018), "Characterisation of the joining zone of serially arranged hybrid semi-finished components", AIP Conference Proceedings Vol. 1960 No. 1, P. 040002. https://doi.org/10.1063/1.5034856

Box, G. and Draper, N. (2007), Response Surfaces, Mixtures, and Ridge Analyses, second edition, John Wiley and Sons, Inc., New Jersey. https://doi.org/10.1002/0470072768

Breitkopf, P. and Coelho, R., eds (2010), Multidisciplinary Design Optimization in Computational Mechanics, John Wiley and Sons, Inc., New Jersey. https://doi.org/10.1002/9781118600153

Brockmoeller, T., Gembarski, P. C., Mozgova, I. and Lachmayer, R. (2017), "Design catalogue in a cae environment for the illustration of tailored forming", in 59th Ilmenau Scientific Colloquium, Ilmenau, Germany.

Dynardo GmbH (2018), Methods for multi-disciplinary optimization and robustness analysis, issue March 2, 2018 edn.

Harzheim, L. (2014), Strukturoptimierung, 2. aufl. edn, Verlag EUROPA-LEHRMITTEL, Haan-Gruiten.

Lauter, C. (2014), Entwicklung und Herstellung von Hybridbauteilen aus Metallen und Faserverbundkunststoffen fuer den Leichtbau im Automobil, PhD thesis, University Paderborn.

R. Myers, D. M. and Anderson-Cook, C., eds (2009), Response Surface Methodology, third edition, John Wiley and Sons, Inc.

Roper, S., Li, D., Florea, V., Woischwill, C. and Kim, I. Y. (2018), “Multi-material topology optimization: A practical approach and application", in WCX World Congress Experience, SAE International. https://doi.org/10.4271/2018-01-0110

Saltelli, A., ed. (2008), Global Sensitivity Analysis. The Primer, John Wiley and Sons, Inc.

Siqueira, R., Bibani, M., Mozgova, I. and Lachmayer, R. (2019), "Case-based parametric analysis: A method for design of tailored forming hybrid material component", in Information Systems Architecture and Technology: Proceedings of 39th International Conference on Information Systems Architecture and Technology - ISAT 2018, Springer International Publishing, Cham, pp. 14-28.

Siqueira, R., Mozgova, I. and Lachmayer, R. (2018), “An interfacial zone evolutionary optimization method with manufacturing constraints for hybrid components", Journal of Computational Design and Engineering. Advance Online Publication. https://doi.org/10.1016/j.jcde.2018.10.003

Ullman, D. (2010), The Mechanical Design Process, McGraw-Hill, New York.

\section{ACKNOWLEDGMENTS}

The results presented in this paper were obtained under the umbrella of Collaborative Research Center 1153 "Process Chain for Manufacturing Hybrid High Performance Components by Tailored Forming", preliminary inspection project $\mathrm{C} 2$. The authors would like to thank the German Research Foundation (DFG) and the CRC 1153 for its financial and organizational support, and also Dynardo GmbH for providing the software Optislang. 